\title{
Targeting inflammation to influence cognitive function following spinal cord injury: a randomized clinical trial
}

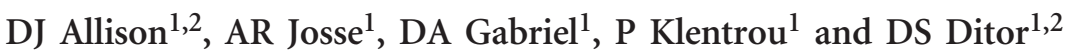

\begin{abstract}
Study design: This study was a randomized, parallel-group, controlled clinical trial.
Objectives: The purpose of this study was to examine the efficacy of targeting inflammation as a means of improving cognitive function in individuals with spinal cord injury.

Setting: Participants were recruited from the Niagara region of Ontario Canada and all testing occurred on-site at Brock University. Methods: Indices of memory and verbal learning were assessed by means of the California Verbal Learning Test (CVLT). Inflammation and concentrations of neuroactive compounds related to the kynurenine pathway were assessed via a number of pro- and anti-inflammatory cytokines, as well as tryptophan, kynurenine and several large neutral amino acids. All assessments were performed at baseline as well as at 1 month and 3 months during a 3-month intervention by means of an anti-inflammatory diet.

Results: Despite a reduction in inflammation, all measures of the CVLT, including list $\mathrm{A}$, trial $1(P=0.48)$, learning slope $(P=0.46)$, long delay free recall $(P=0.83)$, intrusions $(P=0.61)$ and repetitions $(P=0.07)$, showed no significant group $\times$ time interaction.

Conclusion: It may be possible that the reduction in inflammation achieved in the current study was insufficient to induce substantial changes in indices of verbal learning and memory. Alternatively, as these participants likely underwent years of previous chronic inflammation, the underlying hippocampal damage may have negated potential improvements induced by acute reductions in inflammation.
\end{abstract}

Spinal Cord (2017) 55, 26-32; doi:10.1038/sc.2016.96; published online 21 June 2016

\section{INTRODUCTION}

An estimated $10-60 \%$ of individuals with spinal cord injury (SCI) demonstrate some degree of cognitive impairment. ${ }^{1-4}$ This dysfunction includes memory impairment ${ }^{4-6}$ and deficits in verbal learning ${ }^{4-6}$ that would suggest a role for the hippocampus. ${ }^{7}$ Cognitive deficits following SCI are often attributed to concomitant traumatic brain injury and, more recently, chronic hypotension ${ }^{8}$ however, there is evidence to suggest that the chronic inflammation typically demonstrated following $\mathrm{SCI}^{9,10}$ may also contribute to such deficits. Populations with severe cognitive impairment, such as those with Alzheimer's disease, have been consistently shown to demonstrate high levels of inflammation, ${ }^{11}$ and this elevated inflammatory state may indirectly influence the hippocampal function. ${ }^{12}$

Certain proinflammatory mediators such as interferon- $\gamma($ IFN- $\gamma),{ }^{13,14}$ interleukin (IL)- $1 \beta^{14}$ and tumor necrosis factor- $\alpha$ (TNF- $\left.\alpha\right)^{13,14}$ have been shown to possess the ability to upregulate indoleamine 2,3 dioxygenase (IDO), a key enzyme of a critical metabolic pathway known as the kynurenine pathway. This enzyme has a vital role in the metabolism of tryptophan (TRP) and the corresponding production of TRP metabolites such as kynurenine (KYN). ${ }^{12}$ Chronically elevated levels of proinflammatory mediators may therefore result in the chronic upregulation of IDO, resulting in excessive TRP breakdown and the overproduction of TRP metabolites such as KYN. As KYN is a blood-brain barrier (BBB) transportable metabolite, it can move from the periphery and influence concentrations within the brain. Once in the brain, KYN can be further metabolized into non-BBB transportable metabolites such as quinolinic acid (QUIN) and kynurenic acid (KYNA). These metabolites are produced via two distinct branches of the kynurenine pathway. The kynureninenicotinamide adenine dinucleotide (KYN-NAD) branch involves the enzyme kynurenine monooxygenase and is responsible for converting KYN to 3-hydroxykynurenine (3-HK) and QUIN. A second branch, known as the kynurenine-kynurenic acid (KYN-KYNA) branch, involves the enzyme kynurenine aminotransferase and is responsible for the conversion of KYN to KYNA ${ }^{15}$ (see Figure 1). The enzyme kynurenine monooxygenase has been shown to be far less active within the brain than in the periphery, and as such becomes rapidly saturated by elevated levels of KYN. It is therefore likely that, in the presence of high KYN levels within the brain, a shift toward the KYNKYNA branch would occur resulting in the increased production of KYNA. ${ }^{16}$ KYNA is a neuroactive compound that acts as an antagonist of two receptors that have been found to be densely populated on the hippocampus, including the $\alpha$-7-nicotinic acetylcholine $(\alpha 7 \mathrm{nACh})$ receptor and (to a lesser extent) the $N$-methyl-D-aspartate (NMDA) receptor. Each of these receptors has a critical role in synaptic plasticity associated with learning and memory. ${ }^{17}$ The inhibition of $\alpha 7 \mathrm{nACh}$ receptors by KYNA has been shown to result in the reduced release of neurotransmitters such as glutamate, acetylcholine and dopamine, each of which has a critical role in cognitive processes. ${ }^{18-20}$ Therefore, by influencing the neuroactive compounds of the kynurenine pathway, it may be possible for chronically elevated levels of inflammation to indirectly influence cognitive processes.

${ }^{1}$ Department of Kinesiology, Brock University, St Catharines, Ontario, Canada and ${ }^{2}$ Brock-Niagara Centre for Health and Well-Being, St Catharines, Ontario, Canada Correspondence: Dr DJ Allison, Department of Kinesiology, Faculty of Applied Health Science, Brock University, 500 Glenridge Avenue, St Catharines, Ontario, Canada L2S 3A1. E-mail: dallison@brocku.ca

Received 14 January 2016; revised 29 April 2016; accepted 17 May 2016; published online 21 June 2016 


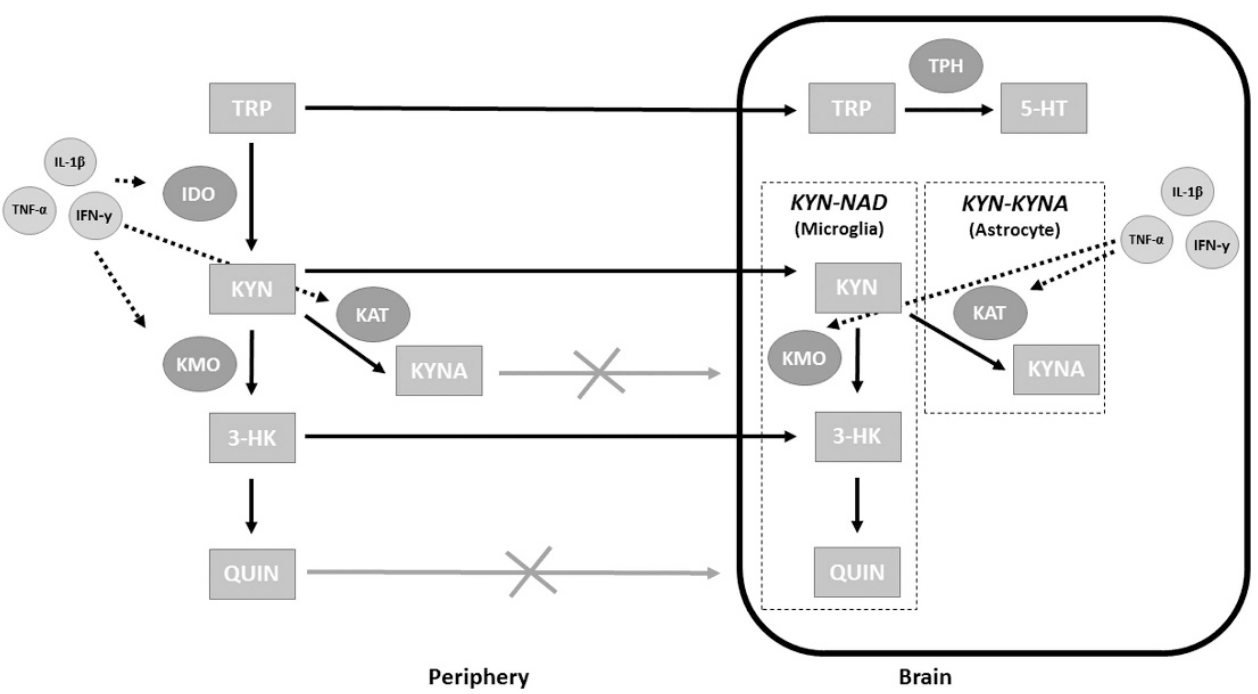

Figure 1 The kynurenine pathway and the influence of proinflammatory mediators. Tryptophan, which is not transported across the BBB for the synthesis of serotonin (5-HT), can be degraded in the periphery by IDO into KYN. KYN may then be metabolized within the periphery or after entering the brain along one of two distinct branches to produce either KYNA or 3-HK and QUIN. The proinflammatory mediators are capable of upregulating the activity of enzymes such as IDO, kynurenine 3-monooxygenase (KMO) and kynurenine aminotransferase (KAT), thereby increasing the production of TRP metabolites. This may result in elevated levels of TRP metabolites with neuroactive properties within the brain. A full color version of this figure is available at the Spinal Cord journal online.

Evidence of this relationship has been demonstrated in animal models whereby elevations in KYNA within the brain have been shown to induce cognitive deficits whether induced indirectly via intraperitoneal administration of $\mathrm{KYN}^{21}$ or via direct intracerebroventricular KYNA infusion. ${ }^{22}$ Furthermore, kynurenine aminotransferase II knockout mice that lack the enzyme responsible for producing KYNA have been shown to have $66 \%$ lower extracellular concentrations of KYNA as well as superior cognitive performance. ${ }^{17}$ In humans, the administration of the noncompetitive NMDA glutamate receptor antagonist, ketamine, has been shown to result in a reduction in verbal declarative memory. ${ }^{23}$ Individuals with Alzheimer's disease have also been shown to exhibit reduced concentrations of TRP and heightened concentrations of QUIN in the periphery. ${ }^{24}$ Furthermore, elevated concentrations of the potent IDO activator TNF- $\alpha$ have been demonstrated in this population, and the administration of the TNF- $\alpha$ antagonist, etanercept, has been shown to result in improved cognitive scores. ${ }^{11,25}$ In addition to pharmaceutical aids, many studies have provided evidence that diet is related to cognitive decline ${ }^{26}$ and that foods high in nutrients with anti-inflammatory properties, such as omega- 3 fatty acids ${ }^{27}$ or flavonoids, ${ }^{28}$ may have a protective effect.

Despite the evidence pertaining to the mechanisms behind such inflammatory-induced cognitive deficits and changes in outcome in both animal models and humans, no study has attempted to define the inflammatory etiology of cognitive deficits in individuals with SCI. Furthermore, very few studies ${ }^{29,30}$ have focused on diet and nutrition following SCI and no study has examined the effects of an antiinflammatory diet following SCI. The purpose of the present study was therefore to assess whether reducing levels of inflammation (via the implementation of an anti-inflammatory diet) in individuals with SCI would be sufficient to induce improvements in memory and verbal learning. It was hypothesized that because of the role of chronic inflammation in the regulation of the kynurenine pathway, reductions in inflammation would result in improved scores for memory and verbal learning.

\section{MATERIALS AND METHODS}

\section{Study design and participants}

This study was performed as a component of a larger clinical trial (Trial Registration: ClinicalTrials.gov identifier: NCT02099890) that included the examination of depression, ${ }^{31}$ neuropathic pain and somatic nerve function. Data pertaining to the change in inflammatory mediators have been previously published. ${ }^{31}$ The study was a randomized, parallel-group, clinical trial. Participant recruitment occurred between September and November 2014. The study intervention was 12 weeks and included testing at baseline and at 1 and 3 months. Participants with various levels and severities of SCI were recruited for participation in the study. Additional inclusion criteria were: (1) age above 18 years, (2) SCI of any level or severity (American Spinal Injury Association A-D) and (3) a duration of at least 2 years after injury. Exclusion criteria included: (1) any contraindications to supplements provided in the study, (2) unstable medical condition within 2 weeks before intervention, (3) pregnancy and (4) breastfeeding. Participant characteristics are shown in Table 1. Twenty individuals ( 10 male and 10 female; age $48.7 \pm 13.9$ years) with chronic (4-37 years after injury) SCI (C2-L4; American Spinal Injury Association Impairment Scale A-D) were recruited for participation in the study. Twelve participants were randomly allocated to the treatment group and were placed on the 12-week anti-inflammatory diet intervention, whereas 8 were allocated to the control group and received no intervention. Informed consent was obtained from all participants. The study was registered as a clinical trial and received ethical approval from the Brock University Research Ethics Board as well as the Natural Health Products Directorate of Canada. All data were collected on-site at Brock University and at the Brock-Niagara Center for Health and Well-Being.

\section{Randomization}

Randomization was computer generated by the primary investigator and stratified by participant sex and age using permuted blocks of 2 (male/female) and 3 ( $<40,40-60$ and $>60$ years). Randomization was 3:2 to either the antiinflammatory diet or the control condition (which received no intervention).

\section{Anti-inflammatory diet intervention}

The anti-inflammatory diet intervention focused on the elimination of common food intolerances and inflammation-inducing foods, as well as the introduction of foods and supplements with established anti-inflammatory properties. All participants received similar instructions regarding foods to 
Table 1 Participant characteristics

\begin{tabular}{|c|c|c|c|c|c|}
\hline Participant & Sex & Age & AIS Score & Level of injury & Time since injury (years) \\
\hline \multicolumn{6}{|l|}{ Treatment } \\
\hline 1 & $\mathrm{~F}$ & 44 & $\mathrm{D}$ & C5 & 10 \\
\hline 2 & $\mathrm{M}$ & 58 & B & T10 & 4 \\
\hline 3 & $\mathrm{~F}$ & 62 & $\mathrm{D}$ & L3 & 4 \\
\hline 4 & $\mathrm{~F}$ & 37 & A & T3 & 19 \\
\hline 5 & $M$ & 22 & A & $\mathrm{C7}$ & 5 \\
\hline 6 & $M$ & 67 & C & $\mathrm{C} 2$ & 4 \\
\hline 7 & M & 66 & $\mathrm{D}$ & C5 & 6 \\
\hline 8 & $\mathrm{~F}$ & 44 & A & $\mathrm{C7}$ & 9 \\
\hline 9 & $\mathrm{~F}$ & 65 & $\mathrm{D}$ & T6 & 4 \\
\hline 10 & $\mathrm{~F}$ & 64 & $D$ & C3 & 37 \\
\hline 11 & $M$ & 45 & A & T6 & 28 \\
\hline 12 & $\mathrm{M}$ & 37 & C & $\mathrm{C} 4$ & 23 \\
\hline AVE & - & 51.5 & - & - & 12.8 \\
\hline s.d. & - & 15.3 & - & - & 11.3 \\
\hline \multicolumn{6}{|l|}{ Control } \\
\hline 13 & $\mathrm{~F}$ & 30 & $B$ & C5 & 6 \\
\hline 14 & $\mathrm{~F}$ & 63 & D & L4 & 2 \\
\hline 15 & $M$ & 42 & A & $\mathrm{C5}$ & 6 \\
\hline 16 & $\mathrm{~F}$ & 58 & $\mathrm{D}$ & $\mathrm{C} 5$ & 33 \\
\hline 17 & $M$ & 59 & D & T4 & 4 \\
\hline 18 & $\mathrm{~F}$ & 33 & A & $\mathrm{T} 1$ & 17 \\
\hline 19 & $M$ & 41 & C & $\mathrm{C} 4$ & 22 \\
\hline 20 & $M$ & 36 & A & $\mathrm{C} 5$ & 19 \\
\hline AVE & - & 45.3 & - & - & 13.6 \\
\hline s.d. & - & 12.9 & - & - & 10.9 \\
\hline$P$-value & & 0.38 & & & 0.87 \\
\hline
\end{tabular}

Abbreviations: AIS, ASIA (American Spinal Injury Association) Impairment Scale; AVE, average; $\mathrm{F}$, female; $\mathrm{M}$, male.

consume and avoid. Examples of foods removed from the diet included those with high glycemic indices (such as refined wheat products and refined sugars), common intolerances such as cow's milk and foods that negatively influence cardiovascular health such as hydrogenated oils. Participants also consumed the following daily supplements. Omega-3 (Now Ultra omega-3, Bloomingdale, IL, USA) was taken in softgel form, containing $500 \mathrm{mg}$ eicosapentaenoic acid and $250 \mathrm{mg}$ docosahexaenoic acid, at a dosage of 3 per day. Chlorella (Now chlorella, Bloomingdale, IL, USA) was taken in pill form, containing $1000 \mathrm{mg}$, at a dosage of 6 per day. Antioxidants (CanPrev Antioxidant Network, Toronto, ON, Canada) were taken in pill form, containing $100 \mathrm{mg}$ coenzyme Q10, $200 \mathrm{mg} \mathrm{N}$-acetyl-cysteine, $150 \mathrm{mg}$ mixed tocopherols, $100 \mathrm{mg}$ DL $\alpha$-lipoic acid, $60 \mathrm{mg}$ green tea extract, $5.5 \mathrm{mg}$ zinc and $100 \mu \mathrm{g}$ selenium, at a dosage of 2 per day. Curcumin (AOR Inflanox, Calgary, AB, Canada) was taken in pill form, containing $400 \mathrm{mg}$, at a dosage of 3 per day. A vegetable-based protein powder (Progressive Vegessential, Toronto, ON, Canada) containing $27 \mathrm{~g}$ of protein was taken at a dosage of one scoop each morning. Both the treatment group and the control group were asked to complete a detailed diet record for 7 days at baseline, as well as for 3 days at 1,2 and 3 months in order to establish baseline eating habits and assess compliance throughout the intervention. Food intake was assessed using The Food Processor (ESHA Inc., 2014, version 10.14.2, Salem, OR, USA). Compliance to the specific anti-inflammatory diet was also assessed by a detailed analysis of all diet records. Each food item was categorized as either a 'food to consume', a 'food to avoid' or a 'neutral food' based on the parameters of the diet that participants were instructed to follow. Food was also categorized into servings in accordance with Canada's Food Guide. Therefore, compliance scores were based on standard servings of foods that subjects were instructed to eat vs foods they were instructed to avoid. To account for differences in total energy intake, compliance scores were expressed as a ratio of the servings of foods to consume over the total servings of food (avoid+consume) multiplied by 100 . The percentage compliance was then generated. The treatment group then underwent an information seminar that explained the diet program followed by a one-on-one consultation with nutritionists during which their diet records were reviewed in detail and necessary changes were discussed. Participants received information regarding foods to eat and avoid, a supplement intake schedule and a list of approved recipes. Participants in the treatment group received support via weekly phone calls from members of our research team as well as a monitored online support group whereby participants could share recipes and experiences with one another. Participants in the control group were asked to maintain their current diets throughout the duration of the study.

\section{Measurement of serum inflammatory markers and amino acids}

Blood draws $(20 \mathrm{ml})$ were taken from the antecubital vein of each participant at $1300 \mathrm{~h}$ at each of the 3 testing sessions (baseline and at 1 and 3 months). Participants consumed a standardized meal consisting of one scoop of Vegessential protein powder with water before the blood draw. Following extraction, the whole blood was allowed to clot for $30 \mathrm{~min}$ followed by centrifugation at $1000 \mathrm{~g}$ for $15 \mathrm{~min}$. Serum was extracted and immediately stored at $-80^{\circ} \mathrm{C}$ until later analysis. Inflammatory mediators of interest included the proinflammatory cytokines IL-2, IL-1 $\beta$, IL- 6 , TNF- $\alpha$ and IFN- $\gamma$, the acute-phase protein, C-reactive protein (CRP), as well as the antiinflammatory cytokines IL-4, IL-10 and IL-1RA. Amino acids of interest included tryptophan, kynurenine, phenylalanine, tyrosine and branched-chain amino acids (valine, leucine and isoleucine). This allowed for the assessment of TRP concentrations as well as the TRP/LNAA ratio (an indicator of TRP availability). Kynurenine levels were also analyzed for the assessment of the KYN/TRP ratio (an indicator of IDO regulation). Analysis of pro- and antiinflammatory cytokines was performed in triplicate using the Magpix Multiplex system (EMD Millipore, Billerica, MA, USA) and analyzed using Luminex software (Austin, TX, USA). CRP was analyzed in triplicate and quantified via an enzyme-linked immunosorbent assay (R\&D Systems, Minneapolis, MN, USA). TRP, phenylalanine, TYR, branch chain amino acid and KYN were analyzed in triplicate and quantified via an enzyme-linked immunosorbent assay (Immunodiagnostik, Bensheim, Germany; Labor Diagnostika, Nordhorn, Germany).

\section{Assessment of cognitive function}

Participants were asked to take the California Verbal Learning Test (CVLT) as an indication of verbal learning and memory. The CVLT has been shown to have strong test-retest reliability, ${ }^{32}$ and has been shown to be a valid assessment of measures related to learning and memory in individuals with multiple sclerosis. ${ }^{33}$ This test consisted of 2 word lists, each consisting of 4 categories of 4 words, for a total of 16 words per list. The test began with the learning trial whereby the examiner read aloud the first list (List A) of words. These words were announced at 1-s intervals, in a fixed order, over 5 learning trials. Following each trial, the participant was asked to recall as many words as possible in any order, including those recalled on previous trials. Following these five learning trials, a single trial of an interference list (List B) was presented, and participants were asked to immediately recall as many words as possible from this new list. This list (List B) consisted of two shared categories from List A as well as two unshared categories. Following the presentation and recall of List B, short delay recall of List A was performed whereby participants were asked once again to recall as many words as possible from List A, both freely (with no help from the examiner) and with semantic cues (whereby participants were told the category; for example, types of vegetables). Following a 20-min delay, long delay recall of List A was assessed, once again first freely and then with semantic cues. Finally, a recognition task was performed whereby participants were verbally presented with 44 words consisting of 16 target words (those from List A) as well as 28 distractor words (those from List B and off-list words). Following the presentation of each word, participants were asked to identify whether or not the word was from List A.

Cognitive performance was examined on the basis of the following: (1) scores from trial 1 of List $\mathrm{A}$, as an indication of short-term memory; (2) the learning slope (slope of scores across the initial five trials of List A), as an indication of the rate of learning; (3) scores of long delay free recall, as an indication of longer-term memory; and (4) errors, including the total number of intrusions (incorrect off-list words) and repetitions (both incorrect and correct) across all trials. 
Table 2 Change in inflammatory mediators

\begin{tabular}{|c|c|c|c|c|c|c|c|c|c|}
\hline & \multicolumn{3}{|c|}{ Treatment $(\mathrm{n}=12)$} & \multicolumn{3}{|c|}{ Control $(n=8)$} & \multirow{2}{*}{$\begin{array}{l}\text { 2-Way } \\
\text { ANOVA } \\
\text { (P-value) }\end{array}$} & \multirow{2}{*}{$\begin{array}{c}\text { Mann-Whitney } \\
\text { (P-value) }\end{array}$} & \multirow{2}{*}{$\begin{array}{c}\text { Friedman } \\
\text { (Treat.) } \\
\text { (P-value) }\end{array}$} \\
\hline & Baseline & 1 Month & 3 Months & Baseline & 1 Month & 3 Months & & & \\
\hline $\begin{array}{l}\text { Proinflammatory } \\
\text { composite } \\
(\mathrm{pg} \mathrm{ml}-1)\end{array}$ & $20.3 \pm 34.5$ & $13.1 \pm 23.6^{*}$ & $14.6 \pm 25.2^{*}$ & $9.8 \pm 11.6$ & $15.4 \pm 22.3$ & $15.7 \pm 25.3$ & - & $<0.01$ & 0.04 \\
\hline CRP (ng ml-1) & $4474.7 \pm 3578.9$ & $3822.6 \pm 3749.4$ & $2865.0 \pm 2684.9$ & $2388.1 \pm 2928.1$ & $3074.0 \pm 3026.4$ & $2458.8 \pm 3678.9$ & - & 0.10 & 0.10 \\
\hline IL-2 (pg ml-1) & $21.3 \pm 51.2$ & $15.1 \pm 41.7$ & $17.2 \pm 42.1$ & $1.7 \pm 3.4$ & $2.9 \pm 3.6$ & $2.3 \pm 3.3$ & - & $<0.01$ & 0.23 \\
\hline IL-6 (pg ml-1) & $13.9 \pm 28.2$ & $9.2 \pm 21.3^{*}$ & $9.5 \pm 19.3$ & $9.0 \pm 10.5$ & $13.8 \pm 21.2$ & $13.5 \pm 21.9$ & - & 0.13 & 0.049 \\
\hline IL-1B (pg ml-1) & $0.9 \pm 1.1$ & $0.3 \pm 0.3^{* *}$ & $0.3 \pm 0.2^{* *}$ & $0.3 \pm 0.3$ & $0.4 \pm 0.5$ & $0.3 \pm 0.2$ & - & $<0.01$ & $<0.01$ \\
\hline $\mathrm{TNF}_{-\alpha}\left(\mathrm{pg} \mathrm{ml^{-1 }}\right)$ & $12.5 \pm 3.6$ & $11.8 \pm 5.5$ & $11.2 \pm 4.1$ & $9.8 \pm 3.9$ & $11.3 \pm 6.7$ & $12.9 \pm 10.3$ & 0.10 & - & - \\
\hline $\mathrm{IFN}-\gamma\left(\mathrm{pg} \mathrm{ml^{-1 } )}\right.$ & $52.9 \pm 94.0$ & $31.9 \pm 57.5^{*}$ & $35.0 \pm 68.4^{*}$ & $28.1 \pm 46.8$ & $48.8 \pm 84.6$ & $49.6 \pm 95.3$ & - & $<0.01$ & 0.01 \\
\hline $\begin{array}{l}\text { Anti-inflammatory } \\
\text { composite } \\
\left(\mathrm{pg} \mathrm{ml} \mathrm{m}^{-1}\right)\end{array}$ & $15.7 \pm 13.7$ & $17.2 \pm 15.4$ & $17.3 \pm 19.1$ & $28.8 \pm 28.3$ & $40.1 \pm 44.9$ & $36.3 \pm 39.5$ & - & 0.32 & 1.0 \\
\hline IL-4 (pg ml-1) & $7.5 \pm 20.8$ & $12.4 \pm 23.9$ & $16.2 \pm 38.4$ & $19.8 \pm 37.2$ & $37.4 \pm 83.8$ & $23.8 \pm 46.3$ & - & 0.54 & 0.63 \\
\hline $\mathrm{IL}-10\left(\mathrm{pg} \mathrm{ml^{-1 }}\right)$ & $6.5 \pm 12.9$ & $11.2 \pm 29.7$ & $9.3 \pm 22.0$ & $5.9 \pm 14.4$ & $5.7 \pm 13.7$ & $6.3 \pm 14.6$ & - & 0.96 & 0.50 \\
\hline IL-1RA (pg ml-1) & $33.1 \pm 26.2$ & $27.8 \pm 18.6$ & $26.3 \pm 16.0$ & $60.6 \pm 66.6$ & $77.2 \pm 77.2$ & $78.8 \pm 105.8$ & - & 0.88 & 0.72 \\
\hline
\end{tabular}

Abbreviations: ANOVA, analysis of variance; CRP, C-reactive protein; IFN- $\gamma$, interferon- $\gamma$; IL, interleukin; TNF- $\alpha$, tumor necrosis factor- $\alpha$; Treat., treatment.

All results are shown as mean \pm s.d. $P$-values correspond to group $\times$ time interactions, Mann-Whitney change scores and Friedman scores (for treatment group) respectively.

Proinflammatory composite consists of a composite score averaging IL-2, IL-6, IL-1B, TNF- $\alpha$ and IFN- $\gamma$.

Anti-inflammatory composite consists of a composite score averaging IL-4, IL-10 and IL-1RA.

Adapted from Allison and Ditor. ${ }^{31}$

* Significantly different from baseline with $P$-value $<0.05$

** Significantly different from baseline with $P$-value $<0.01$.

\section{Statistical analysis}

Two-way (group $\times$ time) repeated-measures analysis of variance was performed to investigate possible changes in scores of the CVLT across the three testing sessions (baseline and at 1 and 3 months). All CVLT subtests were calculated as a $T$-score $(M=50 ;$ s.d. $=10)$. Two-way repeated-measures analysis of variance was also performed for the proinflammatory cytokine TNF- $\alpha$ and the amino acids TRP, TYR and KYN and the TRP/LNAA ratio. As the remaining inflammatory mediators and amino acids were not normally distributed, nonparametric analyses were performed. The Friedman test of differences among repeated measures (baseline and at 1 and 3 months) for the treatment group and control was performed. If the Friedman test resulted in a significant value, the Wilcoxon signed-rank test was performed to provide specific information regarding which time points were significantly different from one another. Finally, Mann-Whitney test was performed on change scores (3 months to baseline) between groups to establish whether the change experienced in inflammatory mediators and amino acids significantly differed between groups. These data are expressed as mean \pm s.d. Statistical significance was set at $P \leqslant 0.05$ for all tests.

\section{RESULTS}

All participants from both the treatment and the control group completed the entire 3-month duration of the study and were included in the analysis. No adverse events were reported. The participants' overall compliance to the diet was assessed on the basis of the average of the 3 diet records during the study (1,2 and 3 months). Participants were required to meet a minimum compliance of $70 \%$ to be included in the analysis. One participant completed all three testing sessions but failed to produce the 2-month and 3-month diet record. This participant had a dietary compliance over the first month of $92 \%$. This participant's 1-month diet record was used in place of the missing 3-month record for the purposes of analysis. All other participants completed each of the required diet records, and overall compliance ranged from 70 to $100 \%$, with a mean compliance of $89 \%$. A detailed analysis regarding specific diet compliance data will be presented elsewhere.

\section{Change in inflammatory mediators}

Changes in serum levels of inflammatory mediators are shown in Table 2. When considering a proinflammatory composite score (average of IL-2, IL-6, IL- $1 \beta$, TNF- $\alpha$ and IFN- $\gamma$ ), the Mann-Whitney test indicated that the change scores (3 months to baseline) were significantly different between the treatment group and the control group $(U=13.0, P=0.01)$. The Friedman test showed that there was a statistically significant reduction in the proinflammatory composite scores in the treatment group $\left(\chi^{2}=6.50, P=0.04\right)$, but no significant change in the control group $\left(\chi^{2}=5.25, P=0.07\right)$. The post hoc analysis performed with the Wilcoxon signed-rank test showed significant reductions in the treatment group from both baseline to 1 month and baseline to 3 months $(z=-2.197, P=0.03 ; z=-2.275, P=0.02$, respectively). When analyzing each cytokine separately, the MannWhitney test indicated that the change scores ( 3 months to baseline) were significantly different between the treatment group and the control group for IFN- $\gamma(U=13.0, P=0.01$.), IL-1 $\beta \quad(U=14.0$, $P=0.01)$ and IL-2 $(U=12.0, P=0.01)$ and showed a trend for CRP $(U=27.0, P=0.10)$. The Friedman test showed that in the treatment group there was a statistically significant reduction in IFN- $\gamma\left(\chi^{2}=8.67\right.$, $P=0.01)$, IL-1 $\beta\left(\chi^{2}=17.78, P<0.01\right)$ and IL-6 $\left(\chi^{2}=6.17, P<0.05\right)$, and a trend for CRP $\left(\chi^{2}=4.5, P=0.10\right)$. The Friedman test showed no statistically significant reductions for any inflammatory mediator in the control group. The post hoc analysis performed with the Wilcoxon signed-rank test showed significant reductions in the treatment group for IFN- $\gamma$ from baseline to 1 month and from baseline to 3 months $(z=-2.275, P=0.02 ; z=-2.510, P=0.01$, respectively), significant reductions in the treatment group for IL- $1 \beta$ from baseline to 1 month and baseline to 3 months ( $z=-3.059, P<0.01 ; z=-2.934, P<0.01$, respectively) and a significant reduction in the treatment group for IL- 6 from baseline to 1 month and a trend from baseline to 3 months $(z=-2.275, P=0.02 ; z=-1.726, P=0.08$, respectively). Two-way repeated-measures analysis of variance was performed for the 


\begin{tabular}{|c|c|c|c|c|c|c|c|c|c|}
\hline & Baseline & 1 Month & 3 Months & Baseline & 1 Month & 3 Months & $\begin{array}{c}\text { 2-Way ANOVA } \\
\text { (P-value) }\end{array}$ & $\begin{array}{c}\text { Mann-Whitney } \\
\text { (P-value) }\end{array}$ & $\begin{array}{c}\text { Friedman (Treat.) } \\
\qquad(\mathrm{P} \text {-value) }\end{array}$ \\
\hline $\operatorname{TYR}\left(\mu \mathrm{mol} \mathrm{I}^{-1}\right)$ & $48.2 \pm 17.7$ & $50.5 \pm 15.1$ & $41.5 \pm 15.2$ & $59.4 \pm 17.6$ & $53.7 \pm 15.7$ & $62.2 \pm 18.3$ & 0.11 & - & - \\
\hline PHE $\left(\mu \mathrm{mol} \mathrm{I}^{-1}\right)$ & $36.6 \pm 13.6$ & $36.8 \pm 13.6$ & $33.6 \pm 14.8$ & $42.0 \pm 16.6$ & $40.8 \pm 18.2$ & $46.2 \pm 23.4$ & - & 0.17 & 0.56 \\
\hline BCAA $\left(\mu \mathrm{mol} \mathrm{I}^{-1}\right)$ & $700.2 \pm 462.4$ & $651.3 \pm 351.9$ & $467.9 \pm 198.5$ & $449.7 \pm 105.5$ & $472.2 \pm 112.1$ & $534.3 \pm 159.2$ & - & 0.03 & 0.34 \\
\hline $\mathrm{KYN}\left(\mu \mathrm{mol} \mathrm{I}^{-1}\right)$ & $2.3 \pm 0.7$ & $2.3 \pm 0.6$ & $2.1 \pm 0.4$ & $1.7 \pm 0.4$ & $2.1 \pm 0.3$ & $2.1 \pm 0.4$ & 0.06 & - & - \\
\hline KYN/TRP & $27.0 \pm 11.6$ & $28.5 \pm 10.9$ & $24.0 \pm 6.6$ & $16.8 \pm 4.9$ & $20.3 \pm 4.7$ & $20.6 \pm 6.0$ & - & 0.03 & 0.35 \\
\hline
\end{tabular}

Abbreviations: ANOVA, analysis of variance; BCAA, branch chain amino acid; KYN, kynurenine; LNAA, large neutral amino acid; PHE, phenylalanine; TRP, tryptophan; TYR, tyrosine. All results are shown as mean \pm s.d. P-values correspond to group $\times$ time interactions, Mann-Whitney change scores and Friedman scores (for treatment group) respectively. Adapted from Allison and Ditor. ${ }^{31}$

Table 4 Change in CVLT scores

\begin{tabular}{|c|c|c|c|c|c|c|c|}
\hline & \multicolumn{3}{|c|}{ Treatment $(n=12)$} & \multicolumn{3}{|c|}{ Control $(n=8)$} & \multirow[t]{2}{*}{ P-value } \\
\hline & Baseline & 1 Month & 3 Months & Baseline & 1 Month & 3 Months & \\
\hline \multicolumn{8}{|c|}{ Trial 1, List A free recall } \\
\hline Raw score & $6.1 \pm 1.2$ & $7.2 \pm 1.5$ & $8.3 \pm 1.7$ & $6.0 \pm 1.7$ & $7.0 \pm 2.2$ & $7.3 \pm 2.1$ & 0.48 \\
\hline$t$-score & $50.3 \pm 8.5$ & $50.5 \pm 8.2$ & $52.8 \pm 8.9$ & $49.7 \pm 11.5$ & $49.6 \pm 11.8$ & $47.2 \pm 11.0$ & \\
\hline \multicolumn{8}{|c|}{ Learning slope coefficient } \\
\hline$t$-score & $47.1 \pm 8.2$ & $50.6 \pm 10.4$ & $49.2 \pm 10.9$ & $52.9 \pm 11.8$ & $49.4 \pm 9.6$ & $50.8 \pm 9.0$ & \\
\hline \multicolumn{8}{|c|}{ Long delay free recall } \\
\hline Raw score & $9.8 \pm 3.1$ & $11.2 \pm 3.3$ & $11.8 \pm 2.9$ & $10.6 \pm 3.3$ & $11.6 \pm 2.9$ & $12.1 \pm 4.1$ & 0.83 \\
\hline$t$-score & $48.8 \pm 9.6$ & $49.3 \pm 10.7$ & $49.6 \pm 8.2$ & $51.2 \pm 10.4$ & $50.7 \pm 9.3$ & $50.4 \pm 11.8$ & \\
\hline Raw score & $4.9 \pm 4.3$ & $3.4 \pm 3.7$ & $4.8 \pm 3.7$ & $5.1 \pm 4.5$ & $8.8 \pm 6.3$ & $4.5 \pm 3.5$ & 0.07 \\
\hline$t$-score & $49.8 \pm 9.7$ & $44.6 \pm 7.4$ & $50.4 \pm 10.3$ & $50.2 \pm 10.3$ & $55.4 \pm 12.6$ & $49.7 \pm 9.7$ & \\
\hline
\end{tabular}

Abbreviation: CVLT, California Verbal Learning Test.

All results are shown as mean \pm s.d. $P$-values correspond to group $\times$ time interactions.

* Significantly different from baseline with a $P$-value $\leqslant 0.05$

normally distributed mediator, TNF- $\alpha$, that showed a trend toward a group $\times$ time interaction $(P=0.10$, Cohen's $d=0.12)$.

\section{Change in serum amino acids}

Changes in serum amino acids are shown in Table 3. Two-way repeated-measures analysis of variance was performed for the normally distributed amino acids. There was a significant group $\times$ time interaction for the TRP/LNAA ratio $(P=0.04$, Cohen's $d=0.90)$. The post hoc analysis showed a strong trend toward an increase in TRP/LNAA in the treatment group from baseline to 3 months $(P=0.06)$. The post hoc analysis showed no change in the control group at any time point. KYN showed a trend toward a group $\times$ time interaction $(P=0.06$, Cohen's $d=0.81)$. The Mann-Whitney test was performed on the change scores for nonnormally distributed amino acids. Change scores (3 months to baseline) for the KYN/TRP ratio were shown to be significantly different between the treatment and control groups $(U=20.0, P=0.03)$. The Friedman test was performed to test for significant changes among repeated measures (baseline and at 1 and 3 months) for the treatment group and control group. No significant change in the KYN/TRP ratio was found in the treatment $\left(\chi^{2}=2.09, P=0.35\right)$ or control $\left(\chi^{2}=4.75, P=0.09\right)$ group.

\section{Change in CVLT scores}

Changes in CVLT scores are shown in Table 4. No significant group $\times$ time interactions were observed for List A, Trial 1 free recall $(P=0.48$, Cohen's $d=0.04)$, learning slope coefficient $(P=0.46$, Cohen's $d=0.04)$ or long delay free recall $(P=0.83$, Cohen's $d=0.01)$. When comparing errors, a significant group $\times$ time interaction was observed for total intrusions $(P=0.03$, Cohen's $d=0.17)$, and a trend was observed for total repetitions $(P=0.07$, Cohen's $d=0.14)$. The post hoc analysis for total intrusions revealed a significant improvement for the treatment group from baseline to 3 months $(P=0.05)$, although there was no significant change in the control group at any time point. The significant group $\times$ time interaction 
Table 5 Correlation matrix

\begin{tabular}{|c|c|c|c|c|c|}
\hline & $\begin{array}{c}\Delta \text { List A, } \\
\text { Trial } 1\end{array}$ & $\begin{array}{c}\Delta \text { Learning } \\
\text { slope }\end{array}$ & $\begin{array}{c}\Delta \text { Long delay } \\
\text { free recall }\end{array}$ & $\Delta / n t r u s i o n s$ & $\Delta$ Repetitions \\
\hline \multicolumn{6}{|l|}{ All participants } \\
\hline$\triangle \mathrm{CRP}$ & -0.086 & -0.053 & 0.047 & 0.170 & 0.098 \\
\hline$\Delta \mathrm{IL}-2$ & 0.212 & -0.356 & -0.078 & 0.176 & 0.276 \\
\hline$\Delta \mathrm{IL}-6$ & 0.198 & -0.415 & -0.224 & $0.616^{* *}$ & 0.373 \\
\hline$\Delta \mathrm{IL}-1 \mathrm{~B}$ & -0.027 & -0.089 & 0.013 & 0.161 & 0.011 \\
\hline$\Delta T N F-\alpha$ & -0.078 & -0.219 & -0.212 & $0.690^{* *}$ & 0.289 \\
\hline$\Delta \mathrm{IFN}-\gamma$ & 0.089 & -0.318 & -0.182 & $0.639^{* *}$ & 0.439 \\
\hline$\Delta \mathrm{IL}-4$ & -0.027 & 0.200 & -0.221 & -0.094 & 0.071 \\
\hline$\Delta \mathrm{IL}-10$ & 0.140 & -0.370 & -0.111 & -0.001 & 0.132 \\
\hline$\Delta I$ L-1RA & -0.017 & -0.130 & 0.066 & $0.598^{* *}$ & $0.522 *$ \\
\hline$\triangle T R P / L N A A$ & -0.298 & 0.177 & 0.041 & -0.162 & -0.079 \\
\hline$\Delta K Y N$ & 0.041 & -0.354 & -0.177 & 0.262 & -0.338 \\
\hline$\Delta K Y N / T R P$ & 0.117 & -0.347 & -0.294 & 0.172 & -0.228 \\
\hline
\end{tabular}

Abbreviations: CRP, C-reactive protein; IFN- $\gamma$, interferon- $\gamma$; IL, interleukin; KYN, kynurenine; LNAA, large neutral amino acid; TNF- $\alpha$, tumor necrosis factor- $\alpha$; TRP, tryptophan.

${ }^{\star} P \leqslant 0.05 ;{ }^{* *} P \leqslant 0.01$.

found for intrusions was, however, heavily influenced by 3 outliers, and upon their removal significance was lost $(P=0.61)$.

\section{Correlational analysis}

Pearson's $r$ correlation coefficients for cytokine, amino acid and CVLT data are shown in Table 5. The change in intrusions was found to be significantly correlated with the change in IL-6 $(r=0.616, P<0.01)$, TNF- $\alpha(r=0.690, P<0.01)$, IFN- $\gamma(r=0.639, P<0.01)$ and IL-1RA $(r=0.598, P<0.01)$. The change in repetitions was found to be significantly correlated with the change in IL-1RA $(r=0.522$, $P=0.02$ ).

\section{DISCUSSION}

The present study was the first to examine the influence of reducing chronically elevated levels of inflammation on cognitive function in vivo in humans with SCI. The intervention successfully reduced levels of proinflammatory mediators but did not achieve significant improvements in any measures of learning and memory as assessed by the CVLT. It was hypothesized that a significant reduction in proinflammatory mediators would be sufficient to induce alterations in neuroactive compounds of the kynurenine pathway, resulting in corresponding improvements in memory. Significant group differences were found for the change in the TRP/LNAA ratio (significant group $\times$ time interaction) as well as the KYN/TRP ratio (significant Mann-Whitney test of change scores). The magnitude of change in these ratios, however, did not reach statistical significance in either the treatment or the control group. It may be possible that the significant reductions obtained in proinflammatory mediators were not considerable enough to induce substantial alterations in IDO regulation and therefore did not allow for meaningful changes in the corresponding amino acids. This may explain, in part, the lack of change in scores of memory and verbal learning related to scores of Trial 1, List A, Learning Slope Coefficient and Long Delay Free Recall.

The lack of such changes may also be attributable to previously developed and potentially irreversible hippocampal atrophy. The TRP metabolite $3-\mathrm{HK}$ is able to cross the $\mathrm{BBB}$ from the periphery and induce oxidative damage through the production of reactive oxygen species following an interaction with the enzyme xanthine oxidase. ${ }^{34}$ In addition, 3-HK may be further metabolized once in the brain into the non-BBB transportable metabolite QUIN. QUIN is a potent agonist of NMDA receptors that are densely populated on the hippocampus. Chronically elevated concentrations of QUIN within the brain are therefore capable of inducing excitotoxicity by causing an overactivation of NMDA receptors, resulting in an increased influx of calcium ions and corresponding neuronal damage. ${ }^{35}$ This may also further contribute to the production of free radicals and oxidative stress brought on by 3 -HK. Together, these mechanisms may contribute to hippocampal atrophy stemming from a chronic elevation in inflammatory mediators. Hippocampal volume loss in the form of reduced gray matter density has been demonstrated in individuals with major depressive disorder (also a chronic inflammatory condition) and has been shown to correlate with reduced scores in verbal recognition memory. ${ }^{36}$ As the sample of the current study consisted of individuals with chronic SCI, it may be possible that years of chronic inflammation resulted in hippocampal atrophy that contributed to deficits in memory and verbal learning that could not be attenuated by an acute reduction in inflammation.

Although not the primary focus of the study, the fact that reductions in inflammation were achieved via an anti-inflammatory diet intervention was a novel aspect of the current study. Research related to diet following SCI has been largely neglected, and to our knowledge this is the first study to utilize an anti-inflammatory diet intervention in this population. Despite the lack of improvements in cognitive function, reductions in inflammation are beneficial for a number of other reasons and has implications in other disorders including neuropathic pain and mood disorders ${ }^{31}$ following SCI as assessed as part of the current clinical trial.

Several potential study limitations should be noted. Although a significant reduction in proinflammatory mediators was achieved, it is unclear how large a reduction may be necessary to induce potential changes in cognition. It may be possible that larger reductions could have had a greater influence. In addition, as hippocampal volume was not assessed in the current study, it is not possible to claim that such an influence was responsible for the lack of effect. In terms of generalizability, our sample was quite representative of the SCI population in Canada regarding age and level of injury, ${ }^{37}$ and the levels of inflammation demonstrated were comparable to those previously reported for this population. ${ }^{9,38,39}$ It may be necessary, however, for future studies to evaluate and control for hippocampal volume when assessing populations that have undergone long periods of chronic inflammation.

\section{CONCLUSION}

The present study demonstrated a lack of improvement in the indices of verbal learning and memory despite a significant reduction of proinflammatory mediators in a population with chronic SCI. These results suggest that reductions in inflammation to this magnitude may not be sufficient to induce meaningful alterations in related neuroactive compounds or corresponding changes in cognition. As it is possible that underlying hippocampal atrophy may have contributed to the lack of improvements in most aspects of the test, it will be necessary for future studies to evaluate hippocampal volume when assessing the efficacy of anti-inflammatory interventions for the treatment of cognitive impairment.

\section{CONFLICT OF INTEREST}

The authors declare no conflict of interest. 


\section{ACKNOWLEDGEMENTS}

This study was supported by the Ontario Neurotrauma Foundation. We thank Now, CanPrev, AOR and Progressive for providing the supplements utilized in the dietary intervention.

1 Davidoff G, Morris J, Roth E, Bleiberg J. Cognitive dysfunction and mild closed head injury in traumatic spinal cord injury. Arch Phys Med Rehabil 1985; 66: 489-491.

2 Davidoff G, Thomas P, Johnson M, Berent S, Dijkers M, Doljanac R. Closed head injury in acute traumatic spinal cord injury: incidence and risk factors. Arch Phys Med Rehabil 1988; 69: 869-872.

3 Richards JS, Brown L, Hagglund K, Bua G, Reeder K. Spinal cord injury and concomitant traumatic brain injury. Results of a longitudinal investigation. Arch Phys Med Rehabil 1988; 67: 211-216.

4 Wilmot CB, Cope DN, Hall KM, Acker M. Occult head injury: its incidence in spinal cord injury. Arch Phys Med Rehabil 1985; 66: 227-231.

5 Davidoff G, Roth E, Thomas P, Doljanac R, Dijkers M, Berent S et al. Depression and neuropsychological test performance in acute spinal cord injury patients: lack of correlation. Arch Clin Neuropsychol 1990; 5: 77-88.

6 Davidoff GN, Roth EJ, Haughton JS, Ardner MS. Cognitive dysfunction in spinal cord injury patients: sensitivity of the Functional Independence Measure subscales vs neuropsychologic assessment. Arch Phys Med Rehabil 1990; 71: 326-329.

7 Papanicolaou AC, Simos PG, Castillo EM, Breier JI, Katz JS, Wright AA. The hippocampus and memory of verbal and pictorial material. Learn Mem 2002; 9: 99-104.

8 Wecht JM, Rosado-Rivera D, Jegede A, Cirnigliaro CM, Jensen MA, Kirshblum S et al. Systemic and cerebral hemodynamics during cognitive testing. Clin Auton Res 2012; 22: 25-33.

9 Davies AL, Hayes KC, Dekaban GA. Clinical correlates of elevated serum concentrations of cytokines and autoantibodies in patients with spinal cord injury. Arch Phys Med Rehabil 2007; 88: 1384-1393.

10 Hayes KC, Hull TCL, Delaney GA, Potter PJ, Sequeira KAJ, Campbell K et al. Elevated serum titers of proinflammatory cytokines and CNS autoantibodies in patients with chronic spinal cord injury. J Neurotrauma 2002; 19: 753-761.

11 Tobinick EL, Gross H. Rapid cognitive improvement in Alzheimer's disease following perispinal etanercept administration. J Neuroinflammation 2008; 5: 2.

12 Allison DJ, Ditor DS. The common inflammatory etiology of depression and cognitive impairment: a therapeutic target. J Neuroinflammation 2014; 11: 151.

13 Kato A, Suzuki Y, Suda T, Suzuki M, Fujie M, Takita T et al. Relationship between an increased serum kynurenine/tryptophan ratio and atherosclerotic parameters in hemodialysis patients. Hemodial Int 2010; 14: 418-424.

14 Nisapakultorn K, Makrudthong J, Sa-Ard-lam N, Rerkyen P, Mahanonda R, Takikawa O. Indoleamine 2,3-dioxygenase expression and regulation in chronic periodontitis. J Periodontol 2009; 80: 114-121.

15 Oxenkrug GF. Tryptophan kynurenine metabolism as a common mediator of genetic and environmental impacts in major depressive disorder: the serotonin hypothesis revisited 40 years later. Isr J Psychiatry Relat Sci 2010; 47: 56-63.

16 Bender DA, McCreanor GM. Kynurenine hydroxylase: a potential rate-limiting enzyme in tryptophan metabolism. Biochem Soc Trans 1985; 13: 441-443.

17 Potter MC, Elmer GI, Bergeron R, Albuquerque EX, Guidetti P, Wu H-Q et al. Reduction of endogenous kynurenic acid formation enhances extracellular glutamate, hippocampal plasticity, and cognitive behavior. Neuropsychopharmacology 2010; 35: 1734-1742.

18 Rassoulpour A, Wu H-Q, Ferre S, Schwarcz R. Nanomolar concentrations of kynurenic acid reduce extracellular dopamine levels in the striatum. J Neurochem 2005; 93: $762-765$.
19 Zmarowski A, Wu H-Q, Brooks JM, Potter MC, Pellicciari R, Schwarcz R et al. Astrocyte-derived kynurenic acid modulates basal and evoked cortical acetylcholine release. Eur J Neurosci 2009; 29: 529-538.

20 Wu H-Q, Pereira EFR, Bruno JP, Pellicciari R, Albuquerque EX, Schwarcz R. The astrocyte-derived alpha7 nicotinic receptor antagonist kynurenic acid controls extracellular glutamate levels in the prefrontal cortex. J Mol Neurosci 2010; 40: 204-210.

21 Chess AC, Simoni MK, Alling TE, Bucci DJ. Elevations of endogenous kynurenic acid produce spatial working memory deficits. Schizophr Bull 2007; 33: 797-804.

22 Chess AC, Bucci DJ. Increased concentration of cerebral kynurenic acid alters stimulus processing and conditioned responding. Behav Brain Res 2006; 170: 326-332.

23 Newcomer JW, Farber NB, Jevtovic-Todorovic V, Selke G, Melson AK, Hershey T et al. Ketamine-induced NMDA receptor hypofunction as a model of memory impairment and psychosis. Neuropsychopharmacology 1999; 20: 106-118.

24 Gulaj E, Pawlak K, Bien B, Pawlak D. Kynurenine and its metabolites in Alzheimer's disease patients. Adv Med Sci 2010; 55: 204-211.

25 Tobinick E, Gross H, Weinberger A, Cohen H. TNF-alpha modulation for treatment of Alzheimer's disease: a 6-month pilot study. MedGenMed 2006; 8: 25.

26 Panza F, Solfrizzi V, Colacicco AM, D'Introno A, Capurso C, Torres F et al. Mediterranean diet and cognitive decline. Public Health Nutr 2004; 7: 959-963.

27 Kalmijn S, van Boxtel MP, Ocké M, Verschuren WMM, Kromhout D, Launer LJ. Dietary intake of fatty acids and fish in relation to cognitive performance at middle age. Neurology 2004; 62: 275-280.

28 Spencer JPE. The impact of fruit flavonoids on memory and cognition. Br J Nutr 2010; 104(Suppl 3): S40-S47.

29 Groah SL, Nash MS, Ljungberg IH, Libin A, Hamm LF, Ward E et al. Nutrient intake and body habitus after spinal cord injury: an analysis by sex and level of injury. J Spinal Cord Med 2009; 32: 25-33.

30 Levine AM, Nash MS, Green BA, Shea JD, Aronica MJ. An examination of dietary intakes and nutritional status of chronic healthy spinal cord injured individuals. Paraplegia 1992; 30: 880-889.

31 Allison DJ, Ditor DS. Targeting inflammation to influence mood following spinal cord injury: a randomized clinical trial. J Neuroinflammation 2015; 12: 204.

32 Woods SP, Delis DC, Scott JC, Kramer JH, Holdnack JA. The California Verbal Learning Test-second edition: test-retest reliability, practice effects, and reliable change indices for the standard and alternate forms. Arch Clin Neuropsychol 2006; 21: 413-420.

33 Stegen S, Stepanov I, Cookfair D, Schwartz E, Hojnacki D, Weinstock-Guttman B et al. Validity of the California Verbal Learning Test-II in multiple sclerosis. Clin Neuropsychol 2010; 24: 189-202.

34 Okuda S, Nishiyama N, Saito H, Katsuki H. 3-Hydroxykynurenine, an endogenous oxidative stress generator, causes neuronal cell death with apoptotic features and region selectivity. J Neurochem 2002; 70: 299-307.

35 Braidy N, Grant R, Adams S, Guillemin GJ. Neuroprotective effects of naturally occurring polyphenols on quinolinic acid-induced excitotoxicity in human neurons. FEBS J 2010; 277: 368-382.

36 Shah PJ, Ebmeier KP, Glabus MF, Goodwin GM. Cortical grey matter reductions associated with treatment-resistant chronic unipolar depression. Controlled magnetic resonance imaging study. Br J Psychiatry 1998; 172: 527-532.

37 Noonan VK, Fingas M, Farry A, Baxter D, Singh A, Fehlings MG et al. Incidence and prevalence of spinal cord injury in Canada: a national perspective. Neuroepidemiology 2012; 38: 219-226.

38 Gibson AE, Buchholz AC, Martin Ginis KA. C-Reactive protein in adults with chronic spinal cord injury: increased chronic inflammation in tetraplegia vs paraplegia. Spinal Cord 2008; 46: 616-621.

39 Manns PJ, McCubbin JA, Williams DP. Fitness, inflammation, and the metabolic syndrome in men with paraplegia. Arch Phys Med Rehabil 2005; 86: 1176-1181. 